\title{
Initial Chest Radiographic Findings in Heatstroke Patients
}

\author{
Amr M. Ajlan, MD \\ Radiology Department, King Abdulaziz University Hospital, King Abdulaziz University, Jeddah, Saudi Arabia
}

\begin{abstract}
Objective: To describe imaging-specific findings on the initial chest radiographs in a group of heatstroke patients. $\underline{\text { Materials }}$ and Methods: An experienced radiologist retrospectively evaluated the initial chest radiographs of 15 heatstroke patients from the September 2016 Mecca stampede. The presence of endotracheal intubation, pleural effusions, airspace opacities, interstitial opacities or bronchial wall thickening was recorded. The chest radiographs were also assessed for being unilaterally or bilaterally abnormal. Results: Assessment included eight (53\%) males and seven (47\%) females (average age of 54 +/- slandered deviation 12 years), eight (53\%) of which were intubated. 12 (80\%) radiographs were abnormal; nine (60\%) bilateral. There were five (33\%) cases of pleural effusions. There were two (13\%) cases of ground-glass opacities, six (40\%) cases with atelectasis, eight (53\%) cases of bilateral bronchial wall thickening and three (20\%) cases of bilateral interstitial lung opacities. No pneumothoraces or consolidations were detected. Conclusion: Most heatstroke patients had bilateral nonspecific initial chest radiographic findings. Bronchial wall thickening, interstitial opacities, and partial atelectasis were most encountered while pleural effusions and ground-glass opacities were less frequent. Further studies are required before our results could be generalized.
\end{abstract}

Keywords: Heatstroke, Radiology, CXR, Chest radiograph, Mecca

\section{Introduction}

Heatstroke is a serious condition that occurs from the elevation of core body temperature above $40^{\circ} \mathrm{C}$, resulting from exposure to hot climate with excessive physical activity (i.e. exertional type) or in the absence of it (i.e. classic type) (1). The primary system affected by heatstroke is that of the central nervous system, but multisystem involvement is common as well $(1,2)$. The respiratory system may be involved with heat stroke in various ways (13 ). To the best of our knowledge, the literature describing the initial chest radiographic imaging features of patients with heat stroke is sparse (1-7). In this study, we aimed to evaluate the initial chest radiographic findings in a group of heatstroke patients referred to our care as a result of the September 2015 Mecca stampede(8).

\section{Materials and Methods:}

The initial chest radiographs of 15 heatstroke patients who presented to our tertiary care center as a result of a the September 2015 Mecca stampede (8)during the yearly Islamic pilgrimagewere assessed. All chest radiographs were obtained according to known standard techniques (9). The patients' age, sex and final outcome were also recorded.

An experienced chest imaging fellowship-trained radiologist analyzed the chest radiographs retrospectively. The presence of endotracheal intubation was evaluated. The radiograph was designated as abnormal if lung or pleural abnormalities were present. Pleural abnormalities were defined as pleural effusions or pneumothoraces. The pleural abnormality was assessed for being unilateral or bilateral, if present. Lung abnormalities were divided into airspace opacities, interstitial opacities or bronchial wall thickening. Airspace opacities were defined as consolidations (increased lung density that obscures underlying lung markings), groundglass opacity (increased lung density that is not obscuring the underlying lung markings) or atelectasis (increased lung density that is associated with features of partial, lobar or total volume loss) (10). Bronchial wall thickening was defined as thick tram track or rounded opacities that continue with hilar airways. Interstitial lines other than bronchial wall thickening constituted interstitial opacities. In the presence of any of the defined lung abnormalities, the chest radiograph was designated as unilaterally or bilaterally abnormal.

\section{Results:}

There were eight (53\%) males and seven (47\%) females, with an average age of $54+/$ - slandered deviation 12 years. Eight (53\%) supine and seven (47\%) anteroposterior sitting chest radiographs were obtained. Nine $(60 \%)$ patients were intubated. Two (13\%) patients eventually died.

There were 12 (80\%) abnormal initial chest radiographs; nine $(60 \%)$ with bilateral and three $(20 \%)$ with unilateral involvement. There were five (33\%) cases of pleural effusions; two (13\%) on the left (Figure 1), one (7\%) on the right and two (13\%) bilaterally. No pneumothoraces were detected on any of the patients.

With regards to lung abnormalities, nine (60\%) were bilaterally abnormal, and three (20\%) were unilaterally abnormal. Ground-glass opacities were detected in two (13\%) of patients, and no patients had consolidations. There were six (40\%) cases with atelectasis; one (7\%) case of complete right upper lobe collapse and five (33\%) cases of partial atelectasis. There were eight (53\%) cases of bilateral bronchial wall thickening (Figure 2) and three (20\%) cases of bilateral interstitial lung opacities.

\section{Discussion}

The initial chest radiographs in our heatstroke population were abnormal in $80 \%$ of the cases, with more bilateral than 


\section{International Journal of Science and Research (IJSR) \\ ISSN (Online): 2319-7064 \\ Index Copernicus Value (2013): 6.14 | Impact Factor (2014): 5.611}

unilateral involvement. However, the detected abnormalities were nonspecific, in the form of bronchial wall thickening, interstitial opacities, atelectasis, ground-glass opacities and pleural effusions.

The literature reports that a subset of heatstroke patients may have a normal initial chest radiograph $(4,5,11)$, which was encountered in $20 \%$ of our patients. The majority of chest radiographic findings previously described were contributed to pulmonary edema or acute respiratory distress syndrome (ARDS) $(4,6,7,12-14)$. Of note, the literature on the topic is not imaging-oriented and does not address specific imaging signs on chest radiography.

Atelectatic changes were the most common finding in our group of patients, mostly partialin nature. In concordance with the literature, our cases frequently reveled bronchial wall thinking and interstitial opacities, which may have resulted from cardiogenic or non-cardiogenic pulmonary edema, ARDS or renal impairment (1-7). Bronchial wall thickening may have also resulted from aspiration as well. Pleural effusions were also encountered in our study, potentially due to pulmonary edema or ARDS. Although there were no consolidations in any of our patients, another form of alveolar density, known as ground-glass opacities, was seen in $13 \%$ of cases. The cause of such finding is not entirely clear, but such a findings may be a result of partial atelectasis, infection, aspiration, pulmonary edema or ARDS (15).

Several limitations should be addressed regarding our study. The relatively small number of patients would restrict applying our results on larger cohorts. However, we hope that our effort would be a stimulating initiative towards assessing initial chest radiographic findings on larger numbers of heatstroke patients. Although our study was geared towards describing the radiographic findings according to standardized imaging-specific signs, our understanding of the cause and significance of such findings has not been particularly evaluated in this paper. Addressing this later point in a more subjective manner would be perhaps doable if a larger cohort of patients is assessed in the future. Finally, a better understanding of chest radiographic findings in heatstroke individuals is expected if correlation was performed with computed tomographyorwith findings on follow-up chest imaging.

\section{Conclusion}

The majority of heatstroke patients demonstrate nonspecific findings on initial chest radiography, mostly bilateral. The most prevalent findings were that of bronchial wall thickening, interstitial opacities, and partial atelectasis. Pleural effusions and ground-glass opacities were not frequent while no consolidations were seen. Further studies of a larger number of patients and with temporal imaging follow-up are needed before our results could be generalized.

\section{References}

[1] Epstein Y, Roberts WO. The pathopysiology of heat stroke: an integrative view of the final common pathway. Scand J Med Sci Sports. 2011.

[2] Leon LR, Bouchama A. Heat stroke. Compr Physiol. 2015 Apr;5(2):611-47.

[3] Dahmash NS, Harthi al SS, Akhtar J. Invasive evaluation of patients with heat stroke. Chest. 1993 Apr;103(4):1210-4.

[4] Sithinamsuwan P, Piyavechviratana K, Kitthaweesin T, Chusri W, Orrawanhanothai P, Wongsa A, et al. Exertional heatstroke: early recognition and outcome with aggressive combined cooling--a 12-year experience. Mil Med. 2009 Apr 30;174(5):496-502.

[5] Roberts WO. Exertional heat stroke during a cool weather marathon: a case study. Med Sci Sports Exerc. 2006 Jun 30;38(7):1197-203.

[6] Muniz AE. Ischemic electrocardiographic changes from severe heat stroke. Southern Medical Journal. 2004.

[7] Muñiz AE. Ischemic electrocardiographic changes and elevated troponin from severe heatstroke in an adolescent. Pediatr Emerg Care. 2012 Jan;28(1):64-7.

[8] The Associated Press. Hajj Stampede in September Killed Over 2,400, New Count Finds. The New York Times.

[9] McAdams HP, Samei E, Dobbins J III, Tourassi GD. Recent advances in chest radiography 1 . Radiology. 2006.

[10] Hansell DM, Bankier AA, MacMahon H, McLoud TC, Müller NL, Remy J. Fleischner Society: glossary of terms for thoracic imaging. Radiology. 2008 Mar;246(3):697-722.

[11] Noakes TD, Opie LH. Heatstroke in a "run for fun". Br Med J. 1979 Jul 7;2(6181):52.

[12] Ramos MJG, Valverde FMG, Álvarez CS, Katnich LO, Quirante FP. Fatal heat stroke in a schizophrenic patient. Case Rep Crit Care. 2011 Dec 31;2012:924328_ 8.

[13] el-Kassimi FA, Al-Mashhadani S, Abdullah AK, Akhtar J. Adult respiratory distress syndrome and disseminated intravascular coagulation complicating heat stroke. Chest. 1986 Sep 30;90(4):571-4.

[14] Angus K, Parsons M, Dubrowski A. Heat Stroke: Emergency Medicine Simulation Scenario. Cureus. 2014.

[15] Hewitt MG, Miller WT, Reilly TJ, Simpson S. The relative frequencies of causes of widespread groundglass opacity: a retrospective cohort. Eur J Radiol. 2014 Sep 30;83(10):1970-6. 


\section{International Journal of Science and Research (IJSR)}

ISSN (Online): 2319-7064

Index Copernicus Value (2013): 6.14 | Impact Factor (2014): 5.611

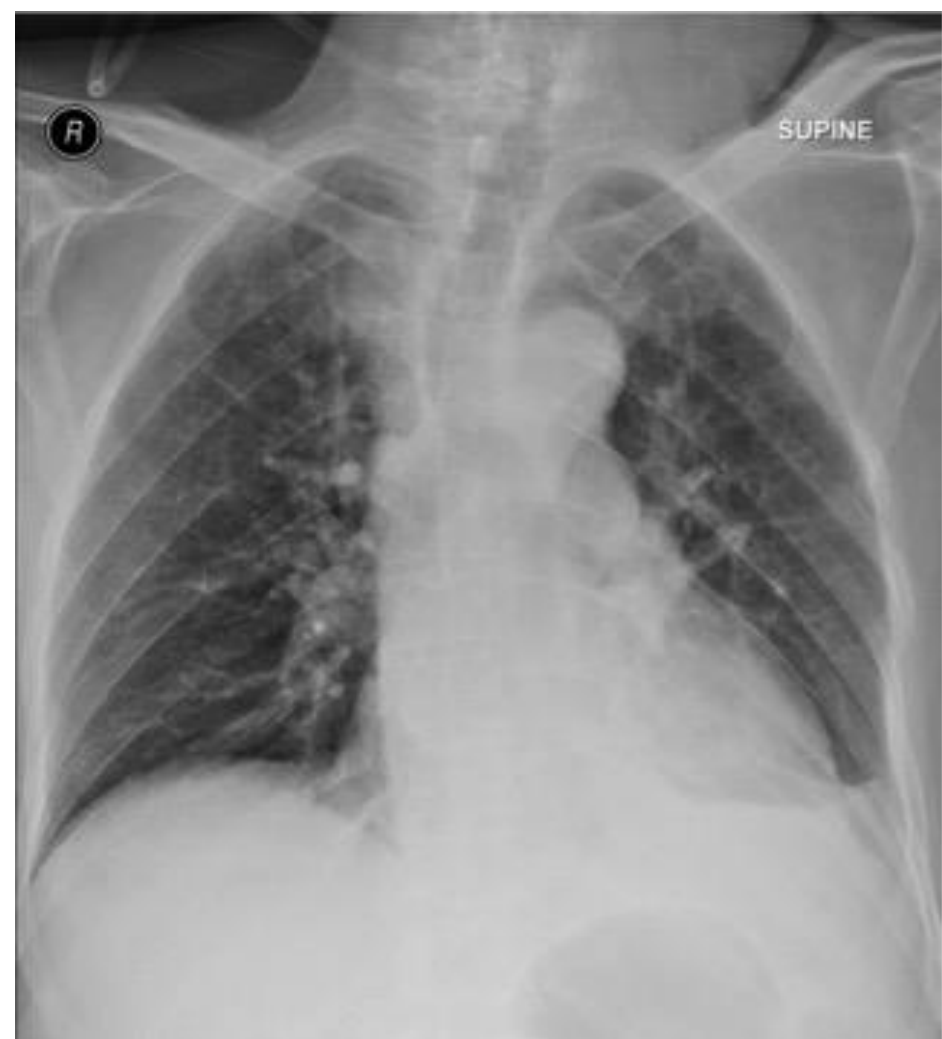

Figure 1: Supine chest radiograph demonstrating a small left pleural effusion.

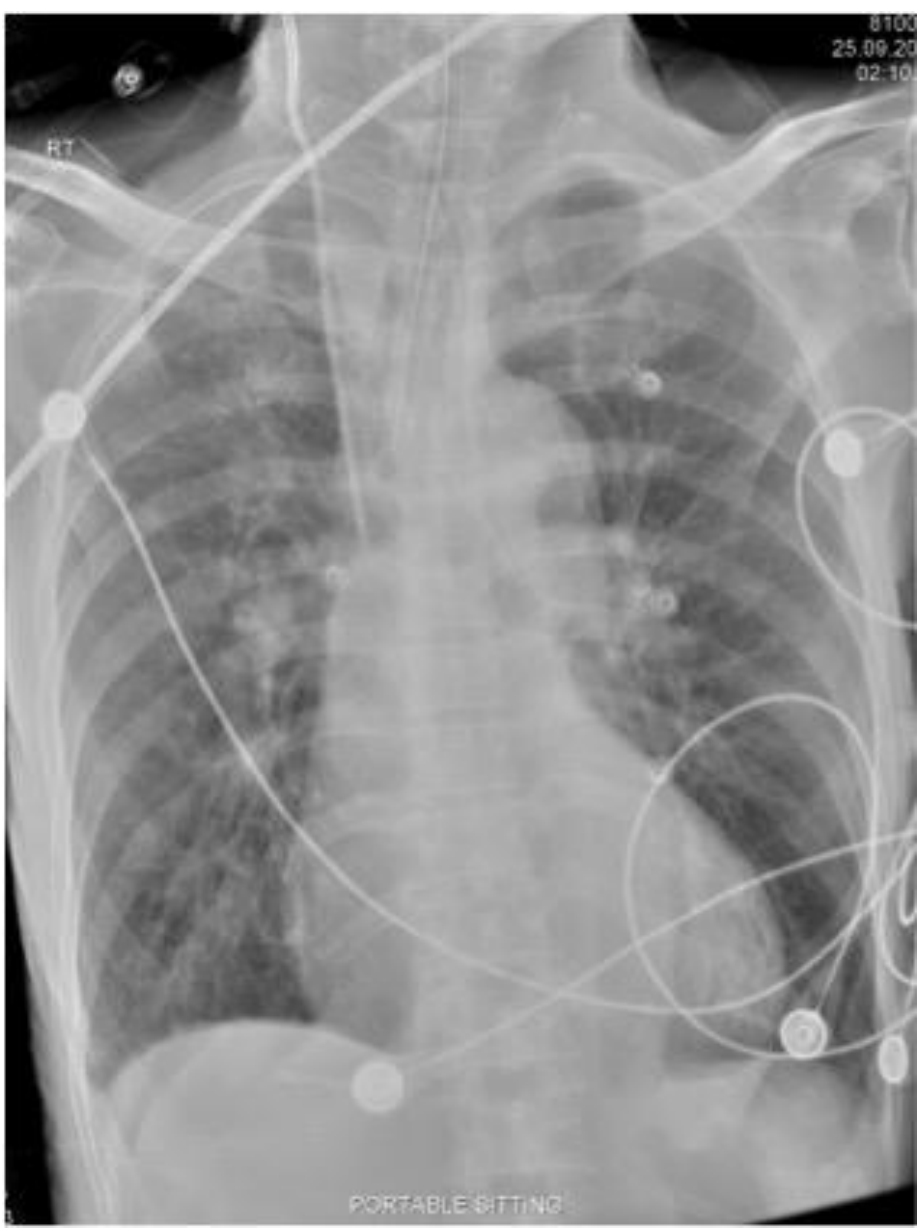

Figure 2: Anteroposterior sitting chest radiograph demonstrating bilateral bronchial wall thickening.

Volume 5 Issue 3, March 2016 www.ijsr.net 